\title{
基于深度学习的自适应水墨画计算美学评估
}

\author{
张佳婧 ${ }^{1)}$ ，于金辉 ${ }^{2}$ ，缪永伟 ${ }^{1)}$ ，彭韧 ${ }^{3)}$ \\ 1) (浙江理工大学信息学院 杭州 310018) \\ 2) (浙江大学 CAD\&CG 国家重点实验室 杭州 310058) \\ 3) (浙江大学计算机科学与技术学院 杭州 310027) \\ (zhangjj@zstu.edu.cn)
}

\begin{abstract}
摘 要: 近几年艺术作品的计算美学评估已成为一个热门的研究方向. 但现有工作主要研究照片和油画, 关于水墨 画的定量审美评估却鲜有尝试. 水墨画通过水墨相调和笔法变化来表现画面，因而在视觉特征、语义特征和审美准则 上与照片和油画有显著不同. 针对此问题，采用深度学习技术，提出一种自适应的水墨画计算美学评估框架. 该框架 首先构建水墨画图像美学评价基准数据集; 然后根据水墨画审美标准提取全局与局部图像块作为多路输人, 并设计 一种多视角并行深度卷积神经网络来提取深度审美特征; 最后基于水墨画的题材查询机制, 构建自适应深度审美评 估模型. 实验结果表明, 文中包含 6 个并行题材卷积组的多视角网络架构相较基础 VGG16 架构有较高的审美评估性 能，提取的深度审美特征明显优于传统手工设计特征，其自适应模型评估结果与人工审美评价之间达到 0.823 的皮尔 森高度显著相关, 且均方误差为 0.161 . 此外, 干扰实验表明, 文中的网络对构图、墨色和纹理 3 个绘画要素较为敏 感. 该研究将不仅为国画计算美学评估提供了一个基于深度学习的参考框架, 而且有助于进一步探索人类审美感知 与水墨画中深度学习特征之间的关系.
\end{abstract}

关键词：深度学习; 水墨画; 计算美学评估; 多视角并行深度神经网络; 自适应模型

中图法分类号: TP391.41 DOI: 10.3724/SP.J.1089.2021.18815

\section{Self-Adaptive Computational Aesthetic Evaluation of Chinese Ink Paintings Based on Deep Learning}

\author{
Zhang Jiajing $^{1)}$, Yu Jinhui ${ }^{2}$, Miao Yongwei ${ }^{1)}$, and Peng Ren ${ }^{3)}$ \\ ${ }^{1)}$ (Department of Information Science and Technology, Zhejiang Sci-Tech University, Hangzhou 310018) \\ 2) (State Key Laboratory of CAD\&CG, Zhejiang University, Hangzhou 310058) \\ ${ }^{3)}$ (College of Computer Science and Technology, Zhejiang University, Hangzhou 310027)
}

\begin{abstract}
Computational aesthetic evaluation of artworks has become an active research direction in recent years. However, current works mainly focus on oil paintings and photographs, there have been few attempts in quantitative aesthetic evaluation of Chinese ink paintings. Chinese ink painting uses ink blended with water and a variation of brushwork to depict picture, which differs significantly from photographs and oil paintings in visual features, semantic features, and aesthetic principles. Aiming at this problem, we propose a framework of self-adaptive computational aesthetic evaluation of Chinese ink paintings based on deep learning technique. Firstly, we build an aesthetic evaluation standard dataset for ink painting images. Secondly, according to aesthetic principles of Chinese ink paintings, we design a multi-view parallel deep neural

收稿日期：2020-11-27; 修回日期：2021-01-27. 基金项目：浙江省自然科学基金(LQ20F020022); 国家自然科学基金(61772463, 61972458); 浙江理工大学科研启动基金(18032115-Y). 张佳婧(1991一), 女, 博士, 讲师, 主要研究方向为计算美学、多媒体计算、深 度学习; 于金辉(1960一), 男, 博士, 教授, 博士生导师, 主要研究方向为计算美学、计算机动画、数字艺术; 缪永伟(1971一), 男, 博 士, 教授, 博士生导师, CCF 会员, 主要研究方向为计算机视觉、数字图像处理; 彭韧(1967一), 男, 博士, 副教授, 硕士生导师, 主要 研究方向为工业设计、媒体与交互设计.
\end{abstract}


network by taking global images and local patches as multi-column inputs to extract deep aesthetic features. Finally, we build a self-adaptive deep aesthetic model of Chinese ink paintings based on subject query mechanism. Experimental results show that, compared with the basic VGG16 architecture, our multi-view network that contains six paralleled subject convolution groups has higher aesthetic evaluation performance, the deep aesthetic features outperform the traditional hand-crafted features, and our proposed self-adaptive model can predict human aesthetic decision with highly significant Pearson correlation of 0.823 , with mean squared error of 0.161 . Moreover, interference experiments show that our network is sensitive to painting factors including composition, ink color, and texture. Our work not only offers a deeply-learned-based reference framework for quantitative aesthetic evaluation of Chinese paintings, but also reveals the relationship between human aesthetic perceptions and deeply-learned features extracted from Chinese ink paintings.

Key words: deep learning; Chinese ink painting; computational aesthetic evaluation; multi-view parallel deep neural network; self-adaptive model

水墨画是中国传统绘画艺术中的典型代表, 通过水墨相调和笔法变化营造墨色干湿浓淡的层 次变化, 有如兼五彩的艺术效果和极高的美学价 值. 传统的水墨画审美评估只能通过鉴赏专家定 性描述, 其结果具有较大的主观不确定性. 此外, 互联网中存储的水墨画图像数量巨大，邀请为数 不多的高水平专家以人工方式对它们逐一评估也 不现实. 如能用计算机高效、自动地对它们进行定 量评估, 将有助于帮助初学者在水墨画学习过程 中对作品进行美感评价, 对水墨画教学有重大指 导意义, 同时对水墨画高级检索和数字国画艺术 馆的推荐展示等领域也有广阔应用前景.

计算美学是最近几年才出现的一个研究方向, 即使计算机能模拟人类视觉系统和审美思维, 并 在相关应用中自动做出可行审美决策的计算方法 研究 ${ }^{[1-2]}$. 目前该工作大部分以照片和西方绘画作 为研究对象, 以图像美感评价数据集为基础 ${ }^{[3-6]}$, 或人工设计视觉特征 ${ }^{[7-13]}$, 或近几年使用深度卷积 神经网络自动提取高层美学特征 ${ }^{[14-23]}$, 而后者在 审美分类与评价任务中取得了优越性能, 但它们 不能完全适用于水墨画的美感定量评估中, 挑战 有如下 3 点.

（1）现有的美感评价数据集大都基于照片或 西方绘画图像构建，而没有公共可用的针对水墨 画美感评估的人工标定方案和图像数据. 需要根 据水墨画审美准则和自身特征从专业角度建立新 的图像美学评价数据集.

（2）水墨画在审美准则上讲究气韵生动、笔墨 神韵、骨法用笔、随类赋彩和经营位置等 ${ }^{[23-26]}$, 这 些评价标准对应高层语义, 抽象程度高, 难以用手
工特征来表示, 易造成语义鸿沟. 此外, 水墨画在 颜色、纹理和构图等视觉特征上与常见艺术形式有 显著不同, 现有方法难以准确和全面地描述水墨 画审美属性.

（3）不同水墨画题材对应不同的审美评估标 准, 例如, 花鸟画通过色彩美抒发情感; 山水画则 强调笔墨技法, 擅用散点透视法造势. 此外, 同一 题材的水墨画根据内容其审美属性也相应变化, 单一审美模型难以适用于所有题材类型. 现有方 法大多依据特定类型场景或先验知识训练独立的 审美模型，具有一定的场景局限性.

为了解决上述问题, 本文以传统水墨画作为 研究对象, 结合美术专业鉴赏理论, 提出一种基于 深度学习的自适应美感定量评估方法. 首先收集 不同题材类型的近现代国画艺术家专业作品及业 余学生作品组成水墨画图像数据, 并基于艺术专 业人员评分构建水墨画美学评估基准数据集, 进 而为美学特征深度学习和模型构建提供了训练和 测试数据. 然后根据水墨画审美特点提取水墨画 全局图像与自适应图像块作为多路输人, 设计一 种多视角并行深度卷积神经网络来提取深度审美 特征, 从而能更好地捕捉和量化水墨画中不同题 材墨色、笔触和构图的审美感知信息. 此外, 本文 将 AlexNet 在 ImageNet 上预训练的模型, 针对水 墨画题材语义进行迁移学习, 进而提取微调后的 网络输出作为视觉表示特征, 并基于题材内容查 询机制，构建自适应的水墨画深度审美评估模型.

该工作是将深度卷积神经网络应用于水墨画 计算美学评估的首次尝试, 为国画定量审美评估 提供了一个基于深度学习技术的参考框架, 是中 
国传统艺术在当下人工智能时代的创新探索, 具 有重要研究意义和科学价值.

\section{1 相关工作}

图像计算美学评估是人工智能和计算机视觉 领域中近几年的一个研究热点. 本文主要针对图 像美学评价基准数据集、图像美感定量评估相关工 作进行介绍与分析.

\section{1 图像美学评价数据集}

在人工主观评价图像美学方面, Luo 等 ${ }^{[3]}$ 构建 了含 17690 张照片的 CUHK-PQ 数据集, 带有二元 审美标签, 并被分组成 7 个场景类别, 即 “动物” “植物” “静物” “建筑” “景观” “人物”和“夜景”. Murray 等 ${ }^{[4]}$ 构建了一个面向图像视觉美学质量分 析与度量的大型图像数据库(aesthetic visual analysis, AVA), 采用在线图像共享评分方法, 数据集包 含 255530 幅图像，每幅图像由人类艺术家会员评 分, 包含 60 多种摄影风格和场景语义标记. Sartori 等 ${ }^{[5]}$ 收集 500 幅艺术博物馆收藏的专业抽象画和 500 幅在线社交网络上传的业余抽象画作为数据 集，并邀请 100 位参观者对每幅作品的第一印象进 行评分, 其中 1 代表非常消极情感，而 7 代表非常 积极情感. Amirshahi 等 ${ }^{[6]}$ 在谷歌艺术项目上收集 了 1600 幅高质量的西方彩色油画，并对这些作品 进行艺术时期、风格和主题等信息标注，建立了 JenAesthetics 数据集. 与上述工作不同, 本文通过 收集艺术专业人员标定数据, 构建了针对水墨画 的人工审美评价数据集.

\section{2 图像美感定量评估}

早期的美学特征人工设计方法研究成果都是 采用人工设计的低层特征来量化实现具体的摄影 技巧或审美准则 ${ }^{[7]}$. 王朝晖等 ${ }^{[8]}$ 通过提取视觉结 构、暗通道和脸部区域特征对肖像照片进行美感评 估. Dhar 等 ${ }^{[9]}$ 通过提取低层特征对构图、场景内容 和自然室外天空光照 3 种高层属性进行评估, 并使 用这 3 种属性来预测照片美感质量和兴趣性. Obrador 等 ${ }^{[10]}$ 提出了一种基于内容的照片美感质量评 估方法，根据不同照片内容将照片分为 7 类，包括 风景、植物、动物、夜景、人像、静物和建筑，并 根据不同类别的评估标准设计相应的主体区域和 视觉特征提取算法. $\mathrm{Su}$ 等 ${ }^{[11]}$ 提出了一种基于“保持 美感特征袋”的风景照片实时视图推荐系统. 然而, 水墨画没有照片里的简洁性、真实度、清晰度、视 彩度、曝光度、景深、模糊效果以及相机基本摄影
技巧等因素影响，也没有肖像照片中的人脸区域 特征, 风景照片中专业的色调组合、室内外、天空 光照等因素，所以上述方法并不完全适用于水墨 画的美感定量评估.

在西方绘画方面, $\mathrm{Li}$ 等 ${ }^{[12]}$ 通过提取颜色分布、 亮度、模糊效果和边缘分布等全局特征, 以及分割 区域的形状信息和颜色对比特征对油画审美视觉 质量进行分类. Sartori 等 ${ }^{[5]}$ 通过提取 LAB 颜色和 SIFT 纹理描述子来区分积极与消极美感的抽象油 画. 但是, 由于油画色彩丰富, 强调光影的表现; 抽象画主要通过形状和颜色以强烈的形式构成来 表达, 而水墨画色彩数量少, 不拘于焦点透视, 不 强调自然界对于物体的光色变化, 更多的是用墨 色浓淡干湿变化和留白来表现层次, 因此, 上述方 法均不能准确和全面地描述水墨画的审美属性.

在水墨画美感评估方面，张佳婧等 ${ }^{[13]}$ 提出一 种基于低层特征的水墨画美感线性回归模型. 但 其只以齐白石花鸟画题材作为数据集，水墨画训 练样本数量很少且缺乏题材和风格的多样性, 未 考虑不同题材内容对美感评估结果的影响, 使得 模型泛化评估能力较弱; 此外其低层手工设计难 以准确量化水墨画高层审美语义.

最近，随着深度学习技术的迅猛发展，一些美 学特征深度学习方法研究工作通过改造用于图像 识别的卷积神经网络 (convolutional neural networks, $\mathrm{CNN})^{[14]}$, 来训练照片标定数据并自动学习 有效的审美特征, 其准确率与人工设计特征相比 有较大提高. $\mathrm{Lu}$ 等 ${ }^{[15]}$ 提出了一种基于深度学习的 照片审美打分模型(rating pictorial aesthetics using deep learning, RAPID), 其中最后一层输出审美二 元分类，并设计成双路视角以共同学习全局和局 部图像特征. Tian 等 ${ }^{[16]}$ 提出一种依赖查询类别的深 度审美模型，但其未考虑局部视角对照片整体美 感评估的影响. Wang 等 ${ }^{[17]}$ 通过设计多场景卷积层 训练深度学习模型来评估照片. Kao 等 ${ }^{[18]}$ 将场景信 息融合到网络中, 提出一种基于多任务关系学习 框架的深度审美模型. $\mathrm{Ma}$ 等 ${ }^{[19]}$ 提出了一种自适应 布局多图像块的深度卷积网络架构来评估照片美 感. Talebi 等 ${ }^{[20]}$ 采用网络输出与真实直方图之间的 距离作为损失函数进行训练, 并提出一种 NIMA 模型来预测人类评估意见分布. Michal 等 ${ }^{[21]}$ 将人 工设计的专业摄影特征与深度美学特征相融合进 行审美预测和分类. 李超等 ${ }^{[22]}$ 以素描作品作为研 究对象, 将深度卷积特征用于素描作品的分类与 评价中. 但是, 上述网络结构大多数基于照片美学 
数据集进行训练, 其模型具有一定的场景局限性. 此外, 传统深度神经网络输人大都根据照片布局 进行随机裁剪, 其输人图像以及网络结构不能准 确地量化水墨画的审美属性. 本文将根据水墨画 自身特点设计网络架构及网络输人, 以达到同时 保留全局和局部细节, 并针对不同题材的水墨画 设计自适应审美模型.

\section{2 本文框架}

图 1 显示了基于深度学习的自适应水墨画计 算审美评估框架. 首先收集 1200 幅专业画家和业 余学生的水墨画作品图像, 并邀请艺术专业人员 进行评分来构建美学评估基准数据集. 然后根据 水墨画审美特点改造基础 VGG16 网络结构，通过 提取水墨画全局图像与自适应多图像块作为多路 输人, 设计一种多视角并行深度卷积神经网络来 提取深度审美特征。最后对于每幅测试图像将 AlexNet 在 ImageNet 上预训练的模型针对水墨画 题材语义进行迁移学习, 进而提取微调后的网络 输出作为视觉表示特征, 并在整个训练集中根据 相似度构建基于题材查询的子训练集，之后在该 子训练集上, 利用深度审美特征训练得到自适应 的水墨画审美评估模型。

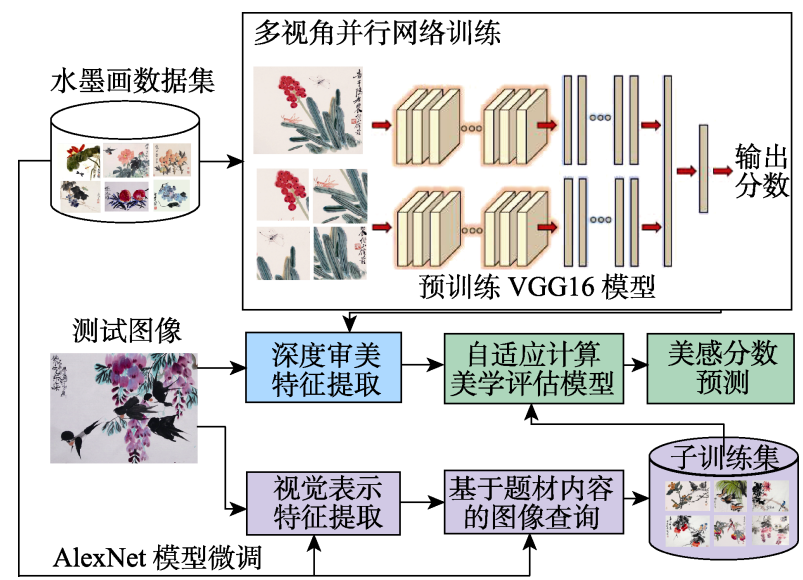

图 1 基于深度学习的自适应水墨画审美评估框架

作为一个基于数据驱动的问题，水墨画审美 特征的深度学习需要依赖训练数据, 因此数据集 的构建就成为关键前提. 下面将详细介绍水墨画 美学评估基准数据集构建方法.

\section{3 水墨画美学评估基准数据集}

在水墨画图像数据收集上，本文拟选取 78 位
近现代著名国画艺术家，如齐白石、李可染、吴昌 硕、王雪涛、潘天寿和张辛稼等的 600 幅专业水墨 画作品, 以及 600 幅业余画家如美术教学中的学生 作品, 其覆盖花鸟草虫、虾蟹、蔬果、走兽、山水 和人物 6 类题材, 将总共 1200 幅水墨画图像作为 数据集, 部分示例如图 2 所示. 其中一部分图像来 自中国美术学院网上公开平台和“台北故宫在线数 据库”, 在类型和大小上进行认真选择, 并以 JPEG 格式下载, 收集了近 100000 幅国画专业作品和数 字文化遗产作品，可以公开访问和下载; 另一部分 图像通过扫描画册而得, 如《中国现代水墨画 $\mathbb{X}$ 水 墨大观》《中国绘画名作欣赏》等知名画家作品集. 之后对这些画作进行基本信息标注，包括画作名 称、作者名称、创作年份、图像大小和题材类型.

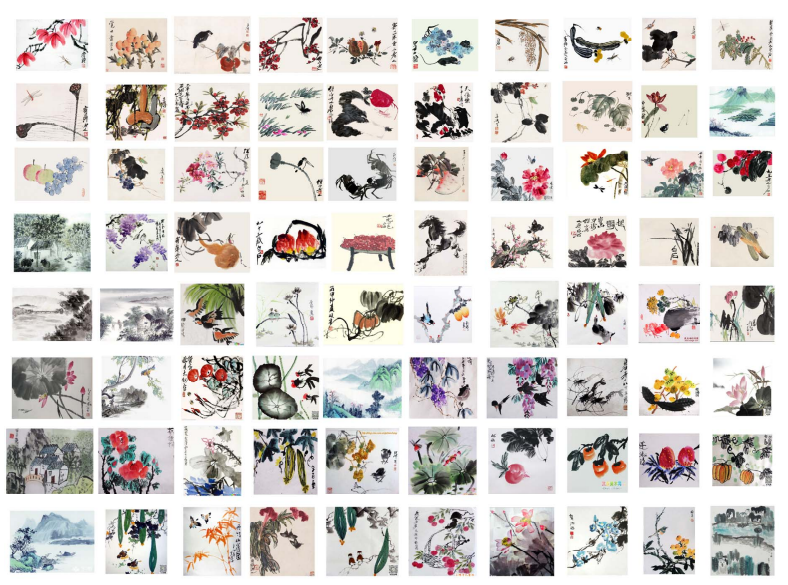

图 2 水墨画数据集示例

在人工评估测试阶段, 本文邀请浙江理工大 学艺术与设计学院、中国美术学院中国画与书法艺 术专业的专家教授、普通教师、国画专业研究生以 及艺术博物馆参观者 (年龄范围是 18 65 岁), 总共 180 名被试者参与评估打分. 根据不同类别标记人 员教育背景和艺术素养的不同，本文设置不同的 权重并计算最终的加权平均分数, 其中专家教授 为 3 , 普通教师为 2 , 研究生和参观者为 1 .

水墨画作为国画的一种重要表现形式，其欣 赏和评价标准来自南朝谢赫在《古画品录》中归纳 的“六法论”，这是千百年来被绘画界和书法界所 公认的, 也是中国古代品评水墨画的重要美学标 准和原则 ${ }^{[26]}$. 基于上述审美标准，本文设计了一 个评估打分页面. 如图 3 所示, 界面上方显示水墨 画作品, 每位测试者要求对每幅作品的以下几个 方面进行评分: “整体美感”用来描述测试者对作品 的整体印象，“墨色搭配”描述画面中色墨交融自然 
度和浓淡层次变化，“笔触变化”描述画面中墨迹干 湿对比度和行笔流畅性, “构图造势”描述画面中物 体和留白的空间组织协调性. 其打分项采用最常 见的 7 级量表进行打分, 范围为 1 7, 7 代表最优分 数, 1 则代表最差分数. 所有图像被随机分成 6 组, 每组 200 幅，每幅作品都会得到 30 个不同测试者 评分. 当所有水墨画作品完成评分后测试结束，每 幅图像的加权平均值作为最终分数, 并用于深度 神经网络模型训练.
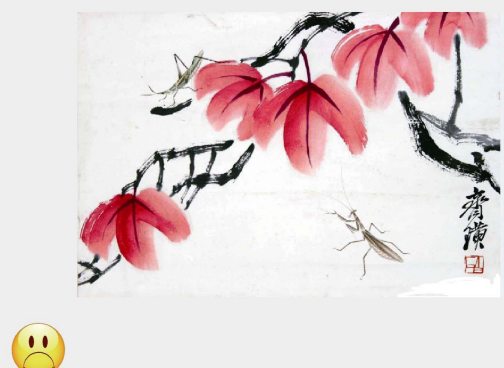

$\begin{array}{lllllll}\text { 整体美感 } \circ 1 & \circ 2 & \circ 3 & \circ 4 & \circ 5 & \circ 6 & \circ 7 \\ \text { 墨色搭配 } \circ 1 & \circ 2 & \circ 3 & \circ 4 & \circ 5 & \circ 6 & \circ 7 \\ \text { 笔触变化 } \circ 1 & \circ 2 & \circ 3 & \circ 4 & \circ 5 & \circ 6 & \circ 7 \\ \text { 构图造势 } \circ 1 & \circ 2 & \circ 3 & \circ 4 & \circ 5 & \circ 6 & \circ 7\end{array}$

用户信息录人 开始评分 上一个 下一个 保存

图 3 人工评估打分页面

\section{4 多视角水墨画深度审美特征提取}

理解并量化水墨画的高层审美语义是弥补低层 视觉特征与高层美学评估间鸿沟的重要环节，而深 度 CNN 在提取高层语义特征方面具有杰出的能力, 其高层输出可以视为高等的审美属性描述子. 因此, 本文根据水墨画审美特点改造基础 VGG16 网络结 构, 设计了多视角并行深度 CNN 来提取审美特征.

\section{1 网络基础架构}

本文将水墨画审美评分任务表示为预测连续 分数的回归建模问题. 将水墨画输人图像表示为 $\boldsymbol{x}_{n}$, 并构造一个深度 $\mathrm{CNN}$ 来自动学习回归映射函 数 $f: \mathbb{R}^{2} \rightarrow \mathbb{R}$, 从而预测美感分数 $\hat{y}_{n}=f\left(\boldsymbol{x}_{n}\right)$. 给 定水墨画训练数据 $\left\{\left(\boldsymbol{x}_{n}, y_{n}\right)\right\}_{n \in[1, N]}$, 其中, $N$ 为训 练集大小, $y_{n}$ 为人工基准评分, $\boldsymbol{W}$ 为网络模型参 数集合. 通过最小化欧几里得回归损失函数来最 优化整个网络,

$$
l(\boldsymbol{W})=\frac{1}{2 N} \sum_{n=1}^{N}\left\|\hat{y}_{n}-y_{n}\right\|_{2}^{2}+\lambda\|\boldsymbol{W}\|_{2}^{2}
$$

其中， $\lambda\|\boldsymbol{W}\|_{2}^{2}$ 表示权重衰减正则化; $\lambda$ 表示正则 强度系数. 为了克服较小水墨画数据集的过拟合 问题, 采用 VGG16 在 ImageNet 数据集上的预训 练模型 ${ }^{[27]}$ 作为基准网络进行迁移学习 ${ }^{[28]}$, 如图 4 所示. 该网络结构包括 13 个卷积层和 3 个全连接 层, 固定前 12 个卷积层结构, 而对其他层进行如 下调整：

Step1. 参考文献[17]中的多场景层设计了一种题 材卷积层来替换原始网络第 13 层, 该题材层由 6 个并行 的卷积网络组构成 (每组 64 个卷积核), 分别用来提取水 墨画中花鸟草虫、虾蟹、蔬果、走兽、山水、人物 6 类 不同题材的审美描述符，该层通过 6 个独立分支与第 12 层相连, 其输出通过平均池化与第 1 个全连接层相连, 从而使得网络针对不同的水墨画题材有较强的自适应 学习能力.

Step2. 用包含 1 个神经元的回归层替换最后一个 全连接层的 1000 维分类概率来预测美感分数, 并使用 欧几里得损失层替换掉最后输出的 softmax 损失层.

Step3. 将前 2 个全连接层的神经元数量从 4096 减 少到 512 和 256 , 从而减少参数数量、防止过拟合, 并有 利于特征级联. 经过修改后, 整个网络基础架构如表 1 所示.

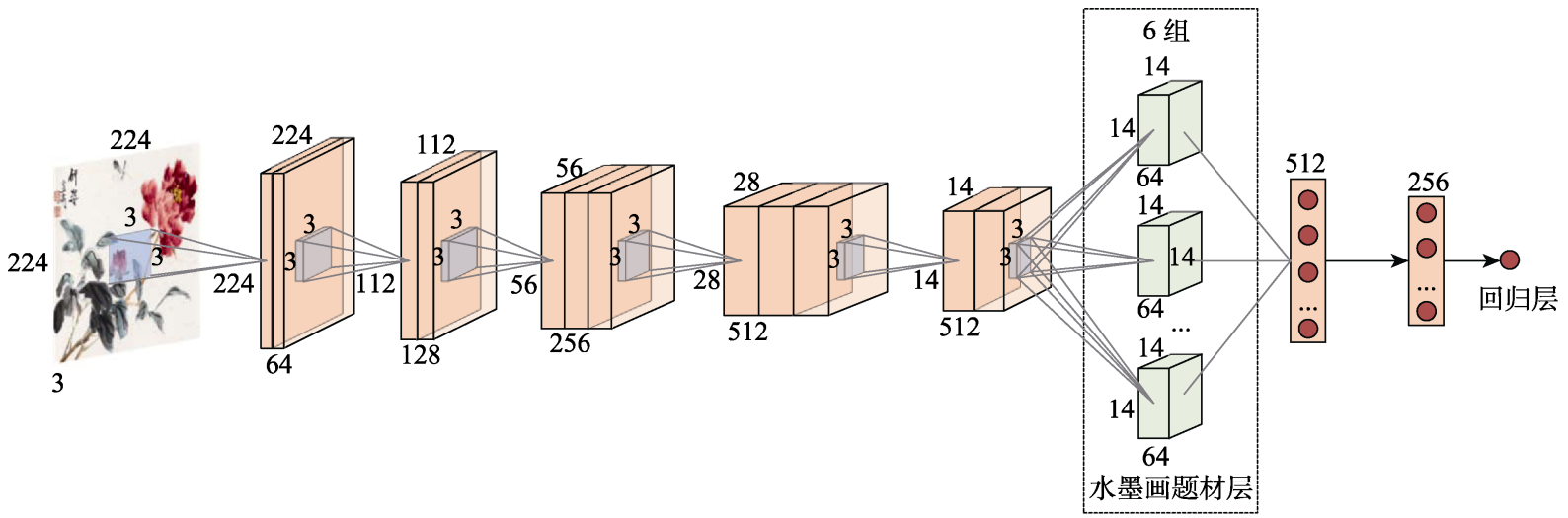

图 4 深度 $\mathrm{CNN}$ 基础架构 
表 1 深度 $\mathrm{CNN}$ 基础架构

\begin{tabular}{lcl}
\hline \multicolumn{1}{c}{ 层数 } & 核大小(步幅) & 特征图大小 \\
\hline conv1-2 & $3 \times 3(1)$ & $224 \times 224 \times 64$ \\
max-pool1 & $2 \times 2(2)$ & $112 \times 112 \times 64$ \\
conv3-4 & $3 \times 3(1)$ & $112 \times 112 \times 128$ \\
max-pool2 & $2 \times 2(2)$ & $56 \times 56 \times 128$ \\
conv5-7 & $3 \times 3(1)$ & $56 \times 56 \times 256$ \\
max-pool3 & $2 \times 2(2)$ & $28 \times 28 \times 256$ \\
conv8-10 & $3 \times 3(1)$ & $28 \times 28 \times 512$ \\
max-poo14 & $2 \times 2(2)$ & $14 \times 14 \times 512$ \\
conv11-12 & $3 \times 3(1)$ & $14 \times 14 \times 512$ \\
conv13(6 组) & $3 \times 3(1)$ & $14 \times 14 \times 64$ \\
fc1 & & $1 \times 1 \times 512$ \\
fc2 & & $1 \times 1 \times 256$ \\
regression & & $1 \times 1 \times 1$ \\
\hline
\end{tabular}

为了能根据不同水墨画题材更有效地提取审 美特征, 本文设计了一种预训练方法来初始化模 型. 在网络参数初始化阶段, 固定 VGG16 模型前 12 个卷积层预训练好的权重, 作为网络中对应层
的初始化参数, 而针对第 13 个卷积层, 使用相应 题材训练数据对每个卷积组进行单独微调, 其相 应网络权重也不断更新. 当所有组都训练完后, 每 组训练权重被并行链接到水墨画题材卷积层来进 行初始化, 从而确保每个题材类别都有自己相应 的神经元激活. 最后 2 个全连接层和回归层参数则 采用随机初始化.

\section{2 多视角并行深度 CNN}

水墨画审美感知需要综合考虑整体和局部视 角，例如山水画在整体构图上讲究“五字法”和“三 远法”，而在局部上讲究留白分布和疏密对比.

通过扩展单路基础网络结构, 本文设计了一 种多视角并行深度 CNN 架构, 如图 5 所示. 首先 根据水墨画审美准则和基本结构提取不同表达形 式的全局图像, 并自适应地选择图像块作为多路 输人; 然后各路输人经过各自 VGG16 网络提取的 特征, 并通过统计聚合结构合并; 最后将输出的全 局和局部特征级联，从而进行审美预测.

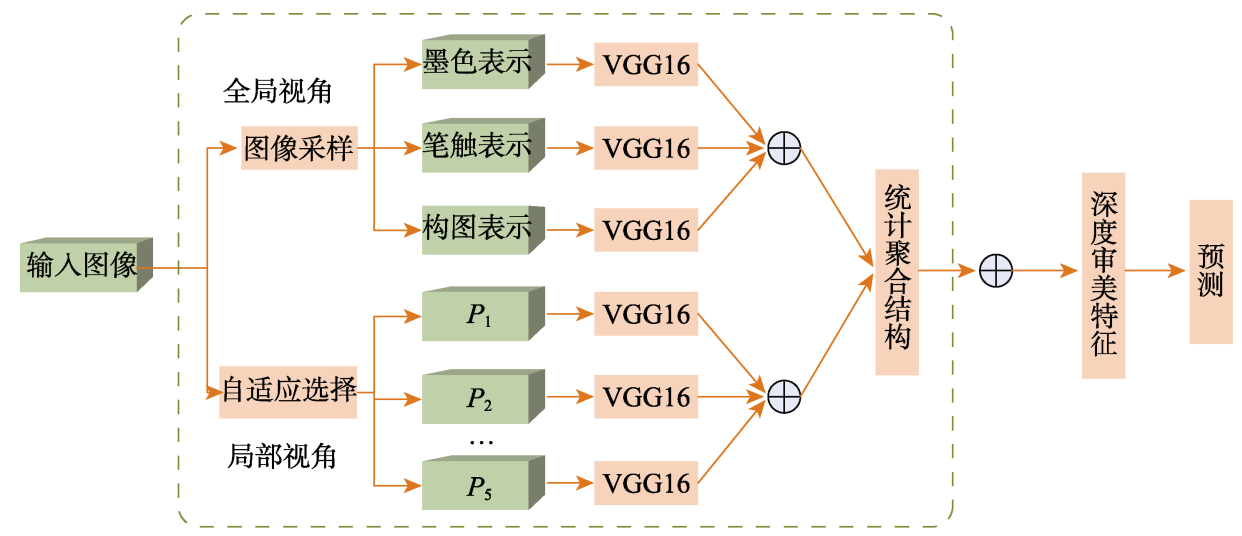

图 5 多视角并行 $\mathrm{CNN}$

\subsection{1 水墨画全局和局部视角输人}

对于全局视角输人，在墨色上，水墨画通过彩 色墨迹笔触、留白之间的对比，以及浓淡灰度变化 来表现. 这里选取水墨画原图、HSV 图的 $H$ 和 $S$ 通道、灰度图作为输人; 在笔触上, 因图像的小波 系数包含丰富的边缘能量信息，能够捕捉水墨画 局部细节运笔信息和典型笔触的纹理特征，本文 利用 Daubechies 第 1 层小波系数矩阵作为纹理输 人. 对于构图，通过计算水墨画的显著图来分析画 面中各个元素的空间分布结构，采用 SLIC 超像素 分割方法 ${ }^{[29]}$ 将原图像分割成若干均匀区域，如图 $6 \mathrm{a}$ 所示. 之后采用 Cheng 等 ${ }^{[30]}$ 提出的基于直方图 对比的方法来计算灰度显著图，如图 $6 \mathrm{~b}$ 所示，其 中较亮区域对应显著度值越高, 其审美视觉上越 重要.

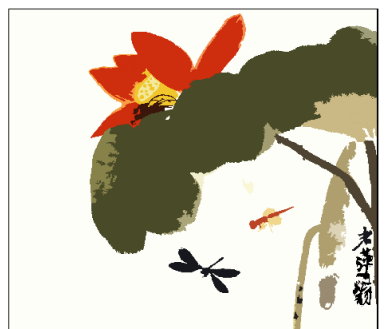

a. SLIC 超像素分割图

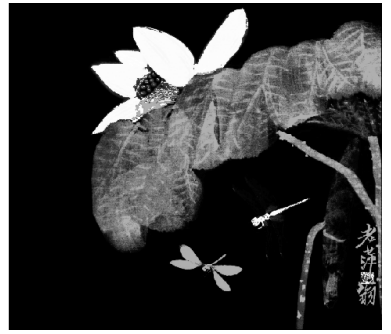

b. 灰度显著图
图 6 水墨画显著图提取

对于局部视角输人，将水墨画表示为裁剪图 像块的集合。与随机裁剪方法 ${ }^{[31]}$ 不同，本文采用 $\mathrm{Ma}$ 等 ${ }^{[19]}$ 提出的自适应策略来提取水墨画中审美感 知信息最多的图像块，其准则有如下几点：(1) 显 著检测能够突出画面中的视觉重要区域，因此使 用显著图去选择图像中最有辨识性和信息量的区 
域; (2) 水墨画中重要的审美细节都要通过不同主 体与背景的关系来表现，所以图像块内要保证模 式多样性; (3) 约束不同图像块之间的空间距离要 进行约束, 从而以保证图像块之间的重叠率尽可 能小. 因此, 将水墨画图像块选择作为一个优化问 题, 定义目标函数 ${ }^{[19]}$

$$
\begin{gathered}
\left\{c^{*}\right\}=\arg \max F\left(S, D_{p}, D_{s}\right) \\
F(\cdot)=\sum_{i=1}^{M} S_{i}+\sum_{i \neq j}^{M} D_{p}\left(\widetilde{N_{i}}, \widetilde{N_{j}}\right)+\sum_{i \neq j}^{M} D_{s}\left(c_{i}, c_{j}\right)
\end{gathered}
$$

其中, $\left\{c_{m}^{*}\right\}_{m \in[1, M]}$ 表示最优化图像块集合每个中心 坐标; $M$ 表示图像块数量； $S_{i}$ 表示每个图像块 $\boldsymbol{p}_{i}$ 的归一化显著度值; $D_{p}(\cdot)$ 和 $D_{s}(\cdot)$ 分别表示模式 距离函数以及欧几里得空间距离函数. 本文采用 多元高斯对每个图像块 $\boldsymbol{p}_{m}$ 模式建模 ${ }^{[19]}$ ，即

$$
\widetilde{N_{m}}=\left\{\left\{N_{e}\left(\mu_{e}, \Sigma_{e}\right)\right\}_{m},\left\{N_{c}\left(\mu_{c}, \Sigma_{c}\right)\right\}_{m}\right\}_{m \in[1, M]}
$$

其中, $\left\{N_{e}\left(\mu_{e}, \Sigma_{e}\right)\right\}_{m}$ 和 $\left\{N_{c}\left(\mu_{c}, \Sigma_{c}\right)\right\}_{m}$ 分别表示该 图像块的笔触边缘分布和浓淡色度分布，其中的 模式距离用来衡量 2 个图像块的差异性. 图 7 展示 了若干采用自适应选择策略提取的图像块结果, 其大小均为 $224 \times 224 \times 3$. 该方法不仅可以有效地选 出最显著区域(如花朵、枇杷和鸟), 而且能捕捉不 同水墨画主体与留白背景之间的模式多样性(如树 干与山水、枝叶与草虫)

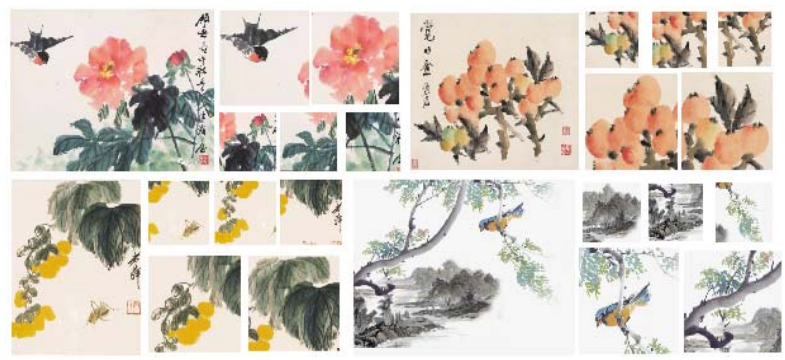

图 7 自适应图像块选择策略提取的图像块示例

\subsection{2 统计聚合结构}

本文采用 $\mathrm{Lu}$ 等 ${ }^{[31]}$ 提出的统计聚合结构来组合 各路经过 VGG16 网络输出的特征. 以局部图像块 为例, 用 $\boldsymbol{D}=\left\{\boldsymbol{d}_{m}\right\}_{m \in[1, M]}$ 表示由 VGG16 网络组输 出的图像块特征集合, 这里 $\boldsymbol{d}_{m}$ 是 $K$ 维向量, $\boldsymbol{T}_{m}=\left\{\boldsymbol{d}_{m k}\right\}_{m \in[1, M]}$ 用来表示所有 $\boldsymbol{d}_{m} \in \boldsymbol{D}$ 第 $k$ 分量集 合, $\oplus$ 用来表示向量级联操作. 统计层由统计函 数集合 $F_{\mathrm{Agg}}=\left\{F_{\mathrm{Agg}}^{u}\right\}_{u \in[1, U]}$ 构成, $U=\left\{F_{\min }, F_{\max }\right\}$. 该集合的输出级联后由全连接层聚合产生一个 $K_{\text {stat }}$ 维特征向量. 整个结构函数 ${ }^{[31]}$ 表示为

$$
g(\boldsymbol{D})=W \times\left(\oplus_{u=1}^{U} \oplus_{k=1}^{K} F_{\text {Agg }}^{u}\left(\boldsymbol{T}_{k}\right)\right)
$$

其中, $W \in \mathbb{R}^{K_{\text {stat }} \times U K}$ 为全连接层参数, 图 8 所示为 $M=4, K=3$ 的统计聚合结构图, 在实验中设置 $M=5, K=256$.

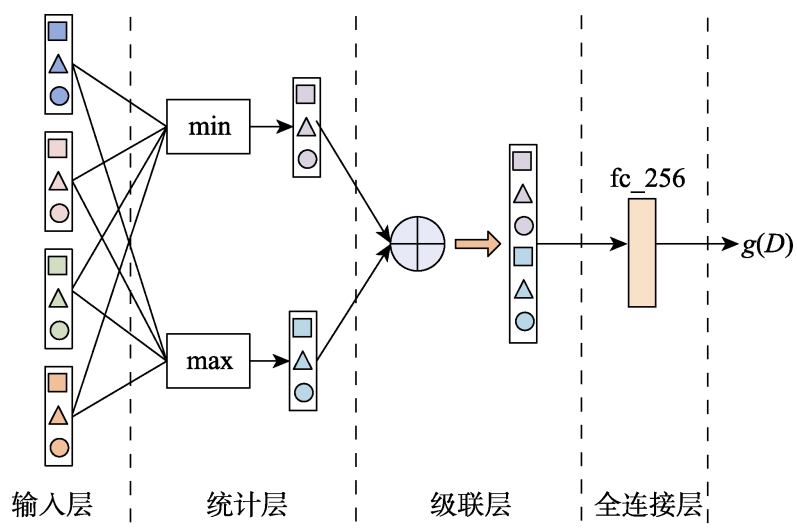

图 8 统计聚合结构图 ${ }^{[31]}$

\subsection{3 训练细节}

首先对各路基准网络进行单独微调, 以此作 为多视角并行网络的初始化权重; 然后分别对全 局视角和局部视角 2 个子聚合网络进行训练, 其中 全局输人为 6 个通道, 局部输人为 5 个通道; 最后 将 2 个子网络输出的 256 维向量进行级联, 并对回 归层权重进行训练, 采用最后输出的 512 维向量作 为水墨画的深度审美特征表示.

\section{5 自适应水墨画深度审美评估模型}

不同题材类型的水墨画作品对应着不同的审 美评估标准，比如山水画侧重于笔墨技法的㿰染 点擦，而花鸟画侧重于色彩点染. 因此，本节利用 第 4 节提取的深度审美特征, 采用 Tian 等 ${ }^{[16]}$ 提出 的照片查询机制, 提出一种基于题材查询的自适 应水墨画审美评估模型，即根据测试图像在训练 集中找到视觉内容或语义相似的图像集合, 进而 训练相应的模型来进行审美预测。

具体地, 每给定一幅测试图像 $\boldsymbol{I}_{q}$, 首先提取 它的视觉表示特征, 本文使用 AlexNet 在 ImageNet 上的预训练模型, 并使用水墨画训练数据微调, 去 除原网络最后一个包含 1000 个神经元的全连接 层, 添加上包含 6 个神经元的全连接层作为分类结 果, 用于代表输出水墨画 6 类题材, 以此适应水墨 画题材分类任务. 然后提取微调后模型的第 7 层全 连接特征 $\boldsymbol{x}_{I}=\left[x_{1}, x_{2}, \cdots, x_{4096}\right]^{\mathrm{T}}$ 来量化表示水墨画 的题材内容. 这种情况下, 测试图片 $\boldsymbol{I}_{q}$ 与训练集 
中图片 $\boldsymbol{I}_{d}$ 间的相似度可计算公式为

$$
\operatorname{Sim}_{\text {visual-CNN }}\left(\boldsymbol{I}_{q}, \boldsymbol{I}_{d}\right)=1-\frac{1}{2} \| \frac{\boldsymbol{x}_{q}}{\left\|\boldsymbol{x}_{q}\right\|_{1}}-\frac{\boldsymbol{x}_{d}}{\left\|\boldsymbol{x}_{d}\right\|_{1} \|_{1}}
$$

之后利用该相似度函数在整个训练集中查找 它的相似图片. 利用这种基于题材检索的返回结 果, 可以构建相应的自适应子训练集

$$
\boldsymbol{L}^{\boldsymbol{I}_{q}}=\left\{\boldsymbol{I}_{d} \mid \boldsymbol{I}_{d} \in \boldsymbol{L} \cap \boldsymbol{I}_{d} \in N\left(\boldsymbol{I}_{d}\right)\right\}
$$

其中, $\boldsymbol{L}$ 为训练集; $N\left(\boldsymbol{I}_{d}\right)$ 为根据视觉表示特征计 算的与测试图像最相似的图片集合，检索过程表 示为 $\mathrm{QDep}_{-} \mathrm{IR}_{\mathrm{CNN}}$. 若干采用 $\mathrm{QDep} \mathrm{IR}_{\mathrm{CNN}}$ 机制 构造的水墨画自适应子训练集如图 9 所示. 接着在 $\boldsymbol{L}^{I_{q}}$ 上利用前面多视角并行网络输出的深度审美特 征，使用支持向量回归(support vector regression, SVR)训练得到自适应审美评估模型, 并利用该模 型预测得到该图像的审美感知分数.
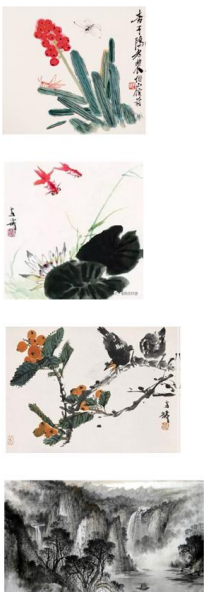

a. 测试图像
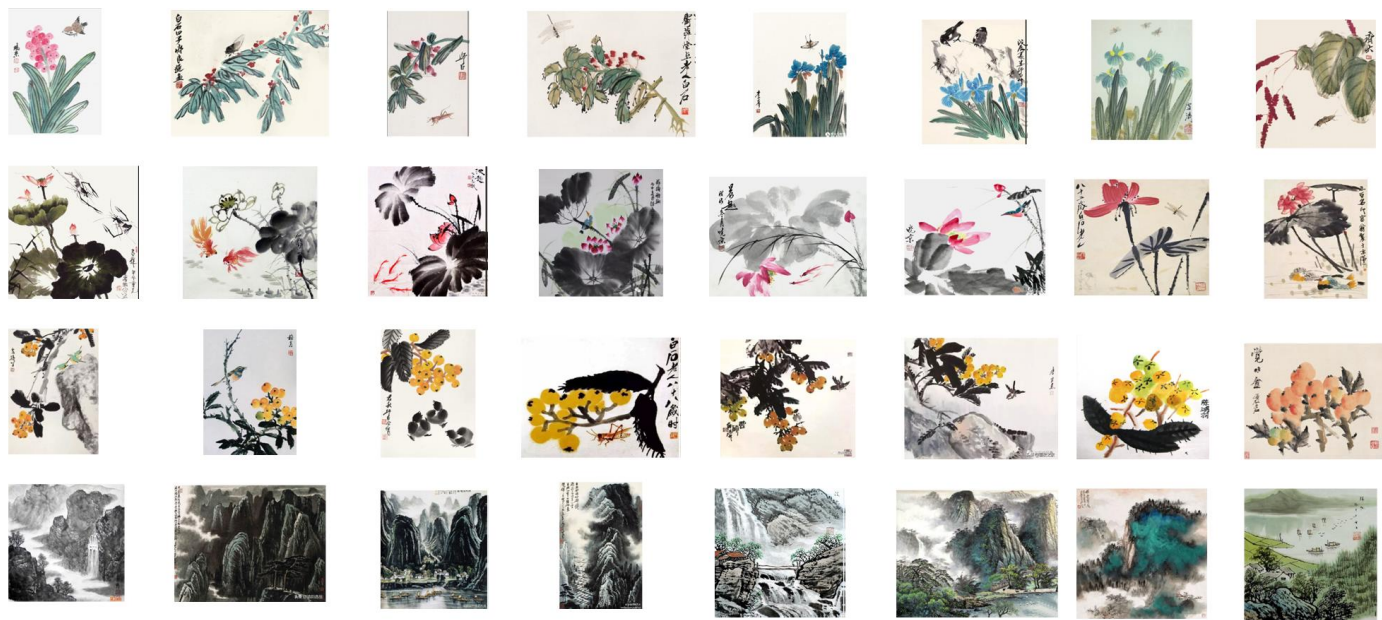

b. 基于题材内容检索返回的前 8 幅图像

图 9 使用 QDep_IR $\mathrm{CNN}_{\mathrm{C}}$ 机制构造的水墨画自适应训练集

\section{6 实验结果与性能分析}

本文所有的网络训练和测试采用 Tensorflow 深度学习框架 ${ }^{[32]}$ 完成, 初始学习率为 0.001 , 每 10 轮缩小 10 倍, 权重衰减率为 $10^{-5}$, 动量为 0.9 . 本 文将 1200 幅作品进行水平映射变化扩大为 2400 幅, 之后将所有作品随机分为 5 组, 每组 480 幅素 描作品; 采用 5 折交叉验证法, 其中 4 组为训练样 本, 1 组为测试样本, 使用随机梯度下降法训练, 5 次实验后的均值为模型最终结果. 在模型性能评 估上, 选取测试集并计算审美模型预测分数与人工 评估分数的平均皮尔森相关系数 $\left(R_{\mathrm{P}} / \mathrm{Sig}\right.$. $)$ 与均方 误差(mean squared error, MSE); 其中, Sig.即 $p$ 值, 代表假设检验中的显著性， $R_{\mathrm{P}}$ 越高且 $\mathrm{Sig} .<0.05$ 时, 表示该模型在统计意义上具有显著性相关; 并且 MSE 越小, 表示预测误差越小, 其模型性能越好. 本节将比较不同基础架构和多视角输人的并行深 度 CNN 性能, 并分析基于题材查询的自适应审美 模型的预测性能.

\section{1 不同架构和视角输入下模型性能比较}

首先以水墨画原图像作为单视角输人, 比较
不同基础架构下单路深度 CNN 模型(single-column deep convolutional neural networks, SCNN)对水墨 画的审美评估性能, 如表 2 所示. 其中, Arc1 表示 原始 VGG16 网络结构, Arc2 表示仅减少了 2 个全 连接层神经元数量而无题材层的 VGG16 网络结 构, $\operatorname{Arc} 3$ 表示将第 13 个卷积层修改为 6 个并行题 材卷积组后的 VGG16 网络结构(本文模型采用的 基础架构). 可以看出, Arc3 架构下的模型相较其 他架构有较高的平均 $R_{\mathrm{P}}(\mathrm{Sig} .<0.05)$ 和较低的平均 MSE，因而对水墨画有较高的审美评估性能. 这 说明在较少网络参数的前提下, 本文网络架构和 迁移学习策略既可以充分地利用预训练模型浅层 的边缘颜色等相关通用审美特征, 又能够结合自 适应的题材卷积组和回归损失层来有效地捕捉不 同水墨画题材的专业审美要素.

表 2 不同基础架构下 SCNN 模型性能比较

\begin{tabular}{lcc}
\hline \multicolumn{1}{c}{ 架构 } & $R_{\mathrm{P}} /$ Sig. & 平均 MSE \\
\hline Arc1 conv13(512)_fc4096_fc4096 & $0.674 / 0.000$ & 0.336 \\
Arc2 conv13(512)_fc512_fc256 & $0.661 / 0.000$ & 0.348 \\
Arc3 conv13(64)_fc512_fc256 & $\mathbf{0 . 7 1 2 / 0 . 0 0 0}$ & $\mathbf{0 . 2 8 5}$ \\
\hline
\end{tabular}

注. 粗体表示本文模型的基础架构及模型性能. 
在此基础上，本节比较不同视角输人下的并 行深度 CNN(multi-view deep convolutional neural networks, MVCNN)模型对水墨画审美感知的预测 性能, 并与之前传统方法进行比较, 如表 3 所示. 为了验证深度学习方法的有效性, 将 MVCNN 与 一些使用人工设计特征的模型, 如 $\mathrm{AVA}^{[4]}$ 和张佳婧 等 ${ }^{[13]}$ 提出的线性回归模型作为基准进行性能比较. 可以看出, 所有深度学习特征的审美评估性能要 显著优于手工设计特征，从而验证了深度学习对 水墨画审美量化评估的有效性. 这种优越的表现 源自深度神经网络能基于人工评分训练数据直接 从原始水墨画中提取高层审美语义特征.

表 3 不同视角输入下 MVCNN 与传统方法性能比较

\begin{tabular}{lcc}
\hline \multicolumn{1}{c}{ 方法 } & $R_{\mathrm{P}} / \mathrm{Sig}$. & 平均 MSE \\
\hline $\mathrm{AVA}^{[7]}(2012)$ & $0.462 / 0.000$ & 0.532 \\
张佳婧等 $^{[13]}(2017)$ & $0.556 / 0.000$ & 0.446 \\
$\mathrm{RAPID}^{[15]}(2014)$ & $0.645 / 0.000$ & 0.371 \\
$\mathrm{DMA}-N e t^{[31]}(2015)$ & $0.654 / 0.000$ & 0.352 \\
$\mathrm{MSDLM}^{[17]}(2016)$ & $0.669 / 0.000$ & 0.344 \\
$\mathrm{SCNN}-A r c 3$ & $0.712 / 0.000$ & 0.285 \\
MVCNN-global & $0.731 / 0.000$ & 0.267 \\
MVCNN-local & $0.782 / 0.000$ & 0.212 \\
MVCNN-hybrid & $\mathbf{0 . 7 9 1 / 0 . 0 0 0}$ & 0.193 \\
\hline
\end{tabular}

注. 粗体表示本文融合全局和局部视角的并行 $\mathrm{CNN}$ 模型 以及预测性能.

此外，本节还将 MVCNN 模型与一些已有的 照片审美评估模型例如 RAPID ${ }^{[15]}$, DMA-Net ${ }^{[31]}$ 和 MSDLM $^{[17]}$ 进行比较, 结果如表 3 所示. 可以看出, MVCNN 模型在水墨画审美预测方面显著优于上 述方法. 该结果进一步验证了 MVCNN 模型的有 效性，特别是自适应选择策略能够有效地提取水 墨画中审美感知信息最多的显著区域，而且能捕 捉不同水墨画主体与留白背景之间的模式多样性, 例如，笔触与留白形成的虚实和疏密对比、色墨对 比、动静对比等.

最后, 本节比较在 $\mathrm{Arc} 3$ 架构下不同视角输人 下的模型预测性能. 其中, SCNN-Arc3 表示将原图 像缩放到 $224 \times 224 \times 3$ 固定大小作为单路输人的 VGG16 模型, MVCNN-global 和 MVCNN-local 分 别表示以全局图像和自适应局部图像块作为输人 的并行网络模型，而 MVCNN-hybrid 表示融合全 局与局部视角输人的并行网络模型. 实验结果表明, 3 种多路并行网络模型 MVCNN-global, MVCNNlocal 和 MVCNN-hybrid 的预测性能要优于单路输人 的 SCNN-Arc3 模型，而 MVCNN-hybrid模型相较其
他单视角模型有较高的平均 $R_{\mathrm{P}}(\mathrm{Sig} .<0.05)$ 和较低 的平均 MSE，因而在水墨画审美评估方面有较高 性能. 这表明模型能够从整体布局信息和局部精 细细节 2 个视角综合提取水墨画审美特征.

\section{2 自适应深度审美模型性能分析}

为了进一步验证自适应模型(第 6 节)预测性能, 将检索过程 QDep_IR $\mathrm{CNN}_{\mathrm{CN}}$ 中的自适应子训练集大小 设为 50 , 并使用 SVR 将第 5 节 MVCNN-hybrid 模型 提取的深度审美特征与人工评估分数进行训练, 其得到的审美评估模型表示 $\mathrm{IR}_{\mathrm{CNN}}+\mathrm{SVR}_{\text {DeepAesth. }}$. 该模型与 MVCNN-hybrid 模型性能进行比较, 如 表 4 所示. 可以看出 $\mathrm{IR}_{\mathrm{CNN}}+\mathrm{SVR}_{\text {DeepAesth }}$ 比 MVCNN-hybrid 有较高的 $R_{\mathrm{P}}$ 和较低的 MSE, 并且 平均 $\mathrm{Sig} .<0.05$, 其模型评估结果与人工审美评分 之间达到 0.823 的皮尔森高度显著相关. 这表明自 适应模型能够有效地捕捉水墨画不同题材内容对 审美标准的影响, 而模型中的深度审美特征在水 墨画计算美学评估方面具有较高的预测性能.

表 4 自适应审美模型与 MVCNN-hybrid 性能比较

\begin{tabular}{lcc}
\hline \multicolumn{1}{c}{ 方法 } & $R_{\mathrm{P}} /$ Sig. & 平均 MSE \\
\hline MVCNN-hybrid & $0.791 / 0.000$ & 0.193 \\
$\mathbf{I R}_{\mathrm{CNN}}+\mathbf{S V R}_{\text {DeepAesth }}$ & $\mathbf{0 . 8 2 3 / 0 . 0 0 0}$ & $\mathbf{0 . 1 6 1}$ \\
\hline
\end{tabular}

注. 粗体表示本文自适应深度审美模型以及预测性能.

一些在审美感知评估分数上呈现对立方向的 水墨画图像比较如图 10 所示. 其中, 顶行作品美 感分数较高, 而底行作品美感分数较低. 图中的数 值表明, 自适应深度审美模型可以很好地描述和 预测水墨画中的美感属性, 并且与人类审美感知 相吻合. 此外, 顶行的水墨画图像与底行相比墨色 浓淡层次变化更加自然, 笔触更加顺畅有序, 干湿 对比更加和谐，并且在画面整体布局上空间感更 好. 由于不同艺术家以不同表现形式灵活地应用 审美准则, 从而形成风格迥异的水墨画艺术. 这些 作品与数据集中大多数相应题材的一般审美准则 相比有较大偏差, 容易被模型评价为美感分数较 低, 但这些著名国画大师的作品因其极高的艺术 价值被人工评价为美感分数较高. 风格抽象的水 墨画表现形式是导致水墨画作品美学评价有较大 误判的主要因素, 图 11 展示了若干评价分数偏差 较大的样本.

此外，本节进一步比较了基于人工设计特征 的线性回归模型等 ${ }^{[13]}$ 、多视角并行深度学习模型 MVCNN-hybrid 和基于题材查询的自适应深度审 


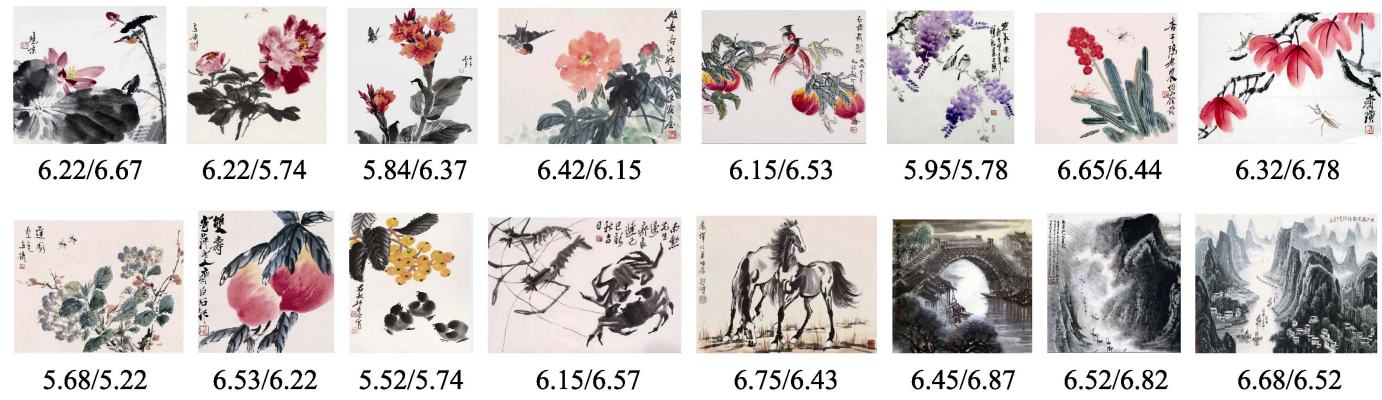

a. 美感高
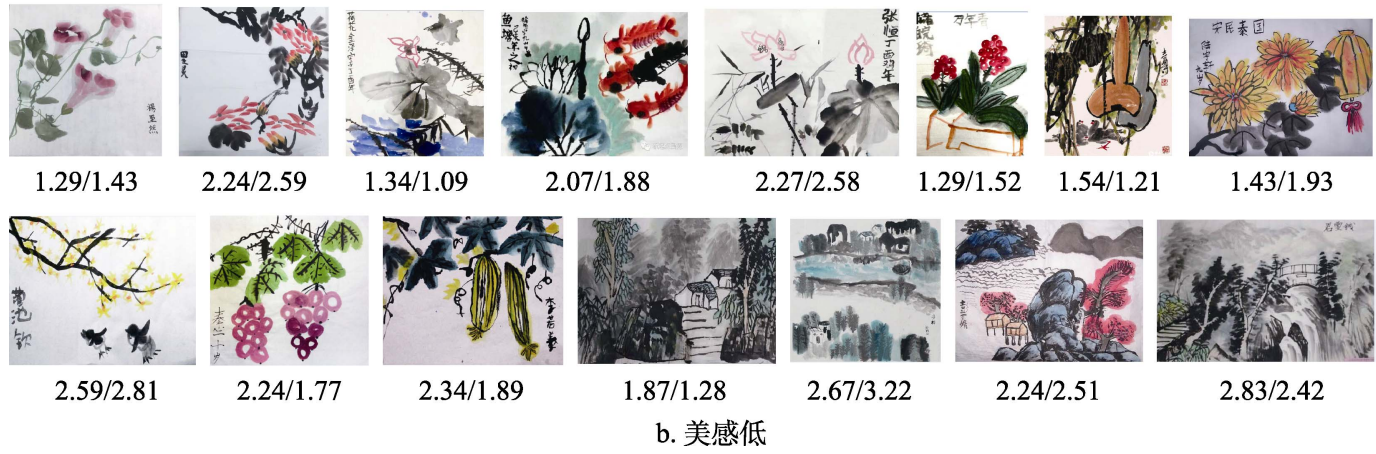

图 10 人工(蓝色)和模型(红色)评估分数比较

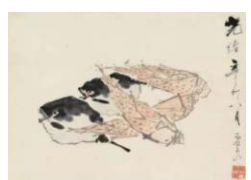

$5.29 / 1.05$

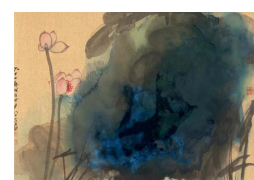

$5.46 / 1.24$

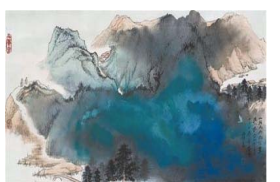

$6.55 / 2.17$

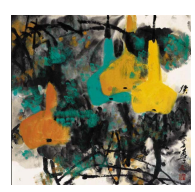

$5.46 / 1.47$

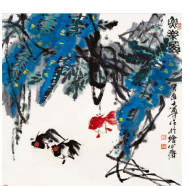

$6.76 / 2.31$

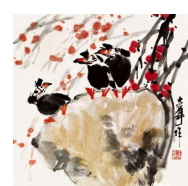

$6.54 / 1.84$

图 11 较大误判的人工(蓝色)和模型(红色)评估分数比较

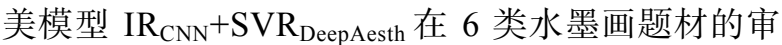
美预测性能, 如图 12 所示. 图中显示 $\mathrm{IR}_{\mathrm{CNN}}+$ $\mathrm{SVR}_{\text {DeepAesth }}$ 在 6 个类别中显著且持续优于其他 2 种模型, 从而进一步证明通过多视角并行 CNN 学 习的审美特征的有效性.

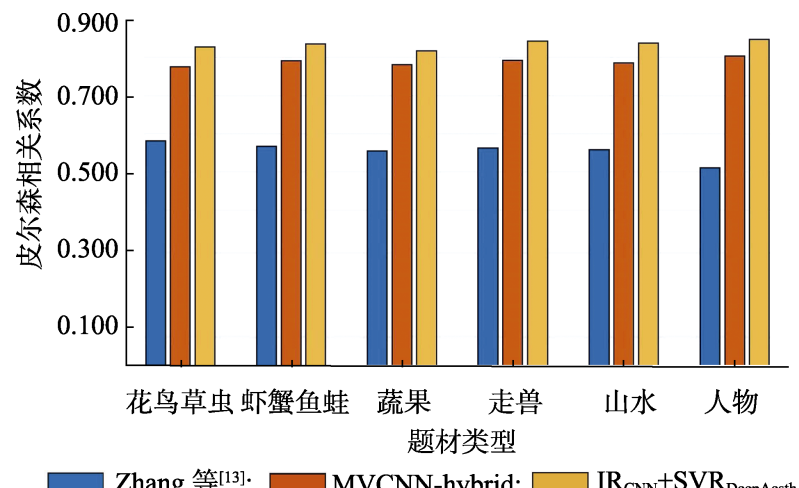

图 12 对不同水墨画题材类别的审美预测性能比较

\section{3 深度审美模型敏感度分析}

为了进一步揭示深度审美模型的可解释性, 这里对构图、墨色、纹理 3 个重要的国画绘画要素 进行实验干扰, 并测试深度 CNN 响应对这些因素
变化的敏感度.

首先选取 100 个大小从 $10 \times 10$ 到 $50 \times 50$ 像素 的小方块, 并在水墨画图像上任意位置进行随机 遮挡，从而对整体布局进行干扰，如图 13 所示. 对 应的性能结果如图 14 中的蓝色线条所示. 可以看 出其模型预测结果与人工评分之间的相关系数随 着布局干扰的增大而快速衰减，这表明深度审美 模型对空间布局较为敏感.

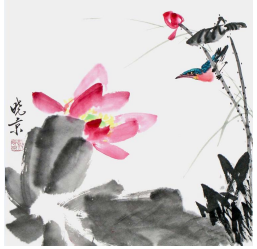

a. 原图

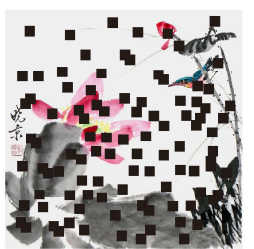

d. $30 \times 30 \times 100$

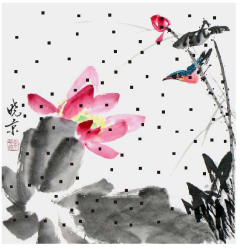

b. $10 \times 10 \times 100$

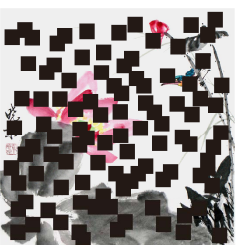

e. $40 \times 40 \times 100$

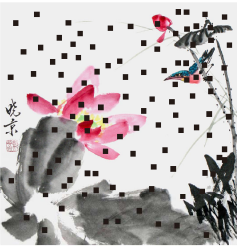

c. $20 \times 20 \times 100$

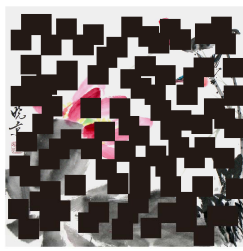

f. $50 \times 50 \times 100$
图 13 对不同大小方块随机遮挡下的水墨画图像 


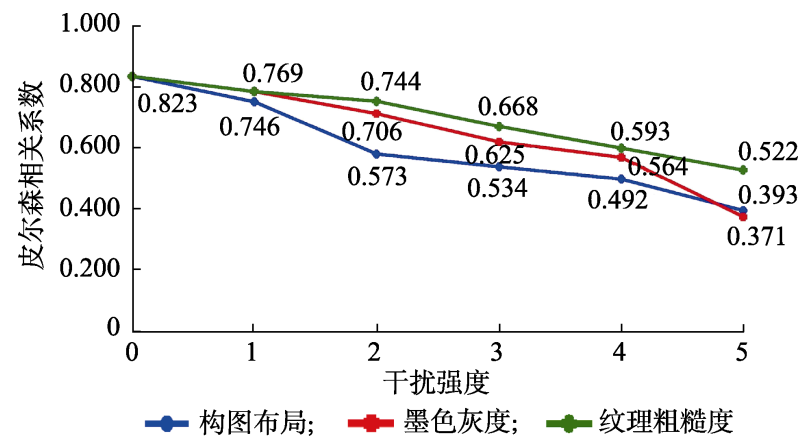

图 14 不同干扰强度下皮尔森相关系数变化

其次，设置不同的灰度系数 $g$ 来对水墨画图 像整体色阶进行干扰, 其范围为 $0 \sim 1$, 越接近 0 , 图像越暗, 如图 15 所示; 对应的性能结果如图 14 中的红色线条所示. 可以看出, 随着灰度系数的下 降, 其相关系数随着色阶干扰的增大而逐渐下降, 表明模型对墨色灰度的层次变化较为敏感.

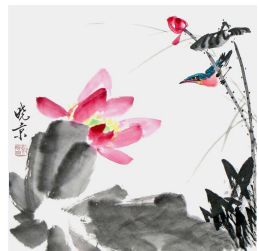

a. 原图

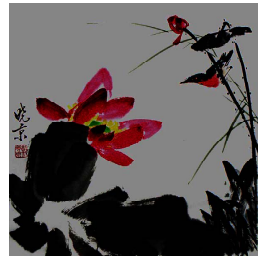

d. $g=0.5$

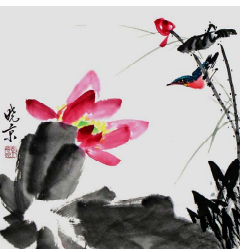

b. $g=0.9$

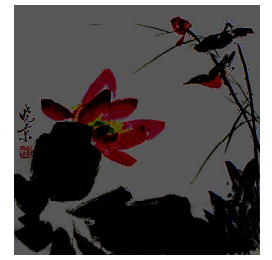

e. $g=0.3$

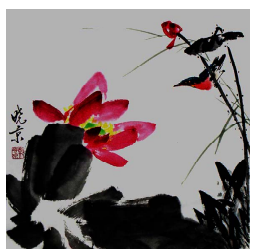

c. $g=0.7$

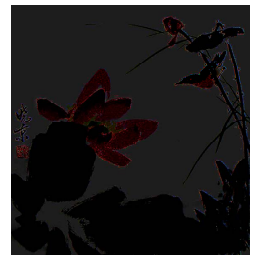

f. $g=0.1$
图 15 不同灰度系数对色阶干扰下的水墨画图像

最后，对水墨画图像添加不同程度的高斯噪 声来对纹理进行干扰, 其方差参数 $s$ 越大, 图像越 粗粘, 如图 16 所示; 对应的性能结果如图 14 的绿

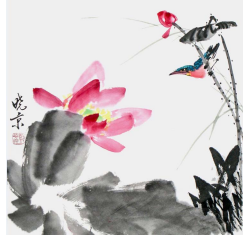

a. 原图

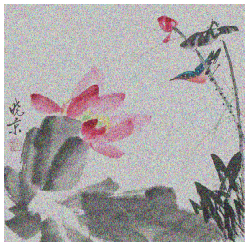

d. $s=0.10$

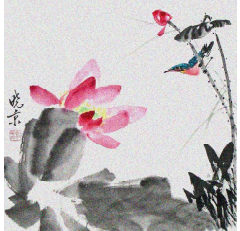

b. $s=0.01$

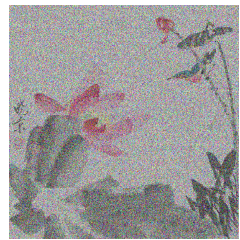

e. $s=0.15$

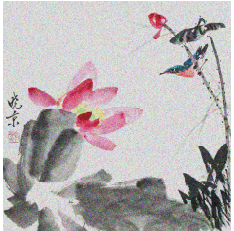

c. $s=0.05$

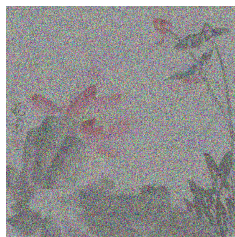

f. $s=0.20$
图 16 不同方差噪声对纹理干扰下的水墨画图像
色线条所示. 可以看出，随着方差的增大，其相关 系数随着噪声增大而逐渐下降, 表明深度审美模 型对笔触纹理较为敏感.

\section{7 结论和未来工作}

本文以水墨画作为对象，提出一种基于深度 学习的自适应计算美学评估框架. 实验结果表明, 自适应深度审美模型评估结果与人工审美评估结 果之间达到 0.823 的皮尔森高度显著相关, 其性能 明显优于传统的基于手工设计特征模型, 证明了 模型的有效性, 并且通过多视角并行 CNN 学习到 的深度审美特征能够准确和敏锐捕捉构图、墨色、 纹理 3 种绘画要素的变化. 该工作为水墨画作品定 量审美评估提供一个基于深度学习技术的参考框 架, 它在一个新的维度上加深了对国画作品审美 感知的理解, 同时为其他绘画艺术形式的计算美 学问题提供新的思路与解决方案.

在未来工作中，将继续探索将手工设计特征 与深度学习特征结合来提高模型对水墨画的审美 评估性能. 此外, 将深度 CNN 扩展到水墨画的多 任务深度学习上, 研究通过水墨画其他高层语义 例如情感意境的预测来更好地辅助水墨画审美评 估任务.

\section{参考文献(References):}

[1] Hoenig F. Defining computational aesthetics[C] //Proceedings of the 1st Eurographics Conference on Computational Aesthetics in Graphics, Visualization and Imaging. Aire-la-Ville: Eurographics Association Press, 2005: 13-18

[2] Fishwick P A. Aesthetic computing[M]. Cambridge: MIT Press, 2006

[3] Luo W, Wang X G, Tang X O. Content-based photo quality assessment[C] //Proceedings of the IEEE International Conference on Computer Vision. Los Alamitos: IEEE Computer Society Press, 2011: 2206-2213

[4] Murray N, Marchesotti L, Perronnin F. AVA: a large-scale database for aesthetic visual analysis[C] //Proceedings of the 25th IEEE Computer Society Conference on Computer Vision and Pattern Recognition. Los Alamitos: IEEE Computer Society Press, 2012: 2408-2415

[5] Sartori A, Yanulevskaya V, Salah A A, et al. Affective analysis of professional and amateur abstract paintings using statistical analysis and art theory[J]. ACM Transactions on Interactive Intelligent Systems, 2015, 5(2): Article No.8

[6] Amirshahi S A, Hayn-Leichsenring G U, Denzler J, et al. JenAesthetics subjective dataset: analyzing paintings by subjective scores[C] //Proceedings of the European Conference on 
Computer Vision. Heidelberg: Springer, 2015: 3-19

[7] Ke Y, Tang X O, Jing F. The design of high-level features for photo quality assessment[C] //Proceedings of the IEEE Conference on Computer Vision and Pattern Recognition. Los Alamitos: IEEE Computer Society Press, 2006: 419-426

[8] Wang Chaohui, Pu Yuanyuan, Xu Dan, et al. Evaluating aesthetics quality in portrait photos[J]. Journal of Software, 2015, 26(S2): 20-28(in Chinese)

(王朝晖, 普园媛, 徐丹, 等. 人像照片的美感质量评价 [J]. 软件学报, 2015, 26(S2): 20-28)

[9] Dhar S, Ordonez V, Berg T L. High level describable attributes for predicting aesthetics and interestingness[C] //Proceedings of the IEEE Conference on Computer Vision and Pattern Recognition. Los Alamitos: IEEE Computer Society Press, 2011: 1657-1664

[10] Obrador P, Saad M A, Suryanarayan P, et al. Towards category-based aesthetic models of photographs[C] //Proceedings of the 18th International Conference on Multimedia Modeling. Heidelberg: Springer, 2012: 63-76

[11] Su H H, Chen T W, Kao C C, et al. Preference-aware view recommendation system for scenic photos based on bag-of-aesthetics-preserving features[J]. IEEE Transactions on Multimedia, 2012, 14(3): 833-843

[12] Li C C, Chen T. Aesthetic visual quality assessment of paintings [J]. IEEE Journal of Selected Topics in Signal Processing, 2009, 3(2): 236-252

[13] Zhang Jiajing, Peng Ren, Wang Jian, et al. Computational aesthetic evaluation of Chinese wash paintings[J]. Journal of Software, 2016, 27(S2): 220-233(in Chinese)

(张佳婧, 彭韧, 王健, 等. 水墨画计算审美评估 [J]. 软件学 报, 2016, 27(S2): 220-233)

[14] Donahue J, Jia Y G, Vinyals O, et al. DeCAF: a deep convolutional activation feature for generic visual recognition[C] //Proceedings of the 31st International Conference on International Conference on Machine Learning. New York: ACM Press, 2014, 32: I-647-I-655

[15] Lu X, Lin Z, Jin H, et al. RAPID: rating pictorial aesthetics using deep learning[C] //Proceedings of the 22nd ACM International Conference on Multimedia. New York: ACM Press, 2014, 457-466

[16] Tian X M, Dong Z, Yang K Y, et al. Query-dependent aesthetic model with deep learning for photo quality assessment[J]. IEEE Transactions on Multimedia, 2015, 17(11): 2035-2048

[17] Wang W N, Zhao M Q, Wang L, et al. A multi-scene deep learning model for image aesthetic evaluation[J]. Signal Processing: Image Communication, 2016, 47: 511-518

[18] Kao Y Y, He R, Huang K Q. Deep aesthetic quality assessmen with semantic information[J]. IEEE Transactions on Image Processing, 2017, 26(3): 1482-1495

[19] Ma S, Liu J, Chen C W. A-Lamp: adaptive layout-aware multi-patch deep convolutional neural network for photo aesthetic assessment[C] //Proceedings of the IEEE Conference on Computer Vision and Pattern Recognition. Los Alamitos: IEEE
Computer Society Press, 2017: 722-731

[20] Talebi H, Milanfar P. NIMA: neural image assessment[J]. IEEE Transactions on Image Processing, 2018, 27(8): 3998-4011

[21] Michal K, Alexander C L, David W M. Leveraging expert feature knowledge for predicting image aesthetics[J]. IEEE Transactions on Image Processing, 2018: 27(10): 5100-5112

[22] Li Chao, Sun Shouqian, Min Xin, et al. Application of deep convolutional features in sketch works classification and evaluation[J]. Journal of Computer-Aided Design \& Computer Graphics, 2017, 27(10): 1898-1904(in Chinese) (李超, 孙守迁, 闵歆，等. 深度卷积特征在素描作品分类与 评价中的应用 $[\mathrm{J}]$. 计算机辅助设计与图形学学报, 2017, 27(10): 1898-1904)

[23] Fan Z B, Zheng X S, Zhang K. Computational analysis and eye movement experiments of white space in Chinese paintings[C] //Proceedings of the IEEE International Conference on Progress in Informatics and Computing. Los Alamitos: IEEE Computer Society Press, 2015: 301-306

[24] Yokochi S, Okada T. Creative cognitive process of art making: a field study of a traditional Chinese ink painter[J]. Creativity Research Journal, 2005, 17(2/3): 241-255

[25] Rawson J. The British museum book of Chinese art[M]. London: The British Museum Press, 2007

[26] Cui Nianhan. Exquisite works of vivid charm-interpretation of the "First of the Six Laws" in Xiehe's collection of ancient paintings[J]. Beauty and Age, 2016, 649(4): 38-39(in Chinese)

(崔念晗. 风神逸品, 气韵生动——解读谢赫《古画品录》中 的 “六法之首” [J]. 美与时代, 2016, 649(4): 38-39)

[27] Krizhevsky A, Sutskever I, Hinton G E. ImageNet classification with deep convolutional neural networks[C] //Proceedings of the 25th International Conference on Neural Information Processing Systems. New York: ACM Press, 2012: 1097-1105

[28] Yosinski J, Clune J, Bengio Y, et al. How transferable are features in deep neural networks[J]. Advances in Neural Information Processing Systems, 2014, 27: 3320-3328

[29] Perazzi F, Krähenbühl P, Pritch Y, et al. Saliency filters: contrast based filtering for salient region detection[C] //Proceedings of the IEEE Conference on Computer Vision and Pattern Recognition. Los Alamitos: IEEE Computer Society Press, 2012: 733-740

[30] Cheng M M, Mitra N J, Huang X, et al. Global contrast based salient region detection[J]. IEEE Transactions on Pattern Analysis and Machine Intelligence, 2015, 37(3): 569-582

[31] Lu X, Lin Z, Shen X, et al. Deep multi-patch aggregation network for image style, aesthetics, and quality estimation[C] //Proceedings of the IEEE International Conference on Computer Vision. Los Alamitos: IEEE Computer Society Press, 2015: 990-998

[32] Abadi M, Barham P, Chen J, et al. TensorFlow: a system for large-scale machine learning[C]//Proceedings of the 12 th USENIX Conference on Operating Systems Design and Implementation. New York: ACM Press, 2016: 265-283 